Astrodynamics Symposium (C1.) Optimization (4.)

Author: Dr. Massimiliano Vasile

University of Glasgow, Glasgow, United Kingdom, mvasile@aero.gla.ac.uk

Mr. Paolo de Pascale

European Space Agency/ESOC, Darmstadt, Germany, Paolo.de.Pascale@esa.int

Dr. Stefano Casotto

CISAS - "G. Colombo" Center of Studies and Activities, Padova, Italy, casotto@pd.astro.it

\title{
ON THE OPTIMALITY OF A SHAPED-BASED APPROACH BASED ON PSEUDO-EQUINOCTIAL ELEMENTS
}

\begin{abstract}
The design of a low-thrust trajectory is typically mathematically formulated as an optimal control problem. Various techniques, developed in the past years, tackle the problem either in a direct way, by transcribing the optimal control problem into a mathematical programming problem, or in an indirect way through the development of the necessary conditions for optimality derived from optimal control theory. Both approaches are not well suited for the very preliminary design phase since both require a first guess solution to be initialized. This paper presents a methodology for the definition of first guesses solutions for optimal trajectory design. This is achieved by a transcription of thrust arcs in terms of pseudo-equinoctial elements. Each element is defined through a parameterised shaping function of the true longitude. By this approach boundary conditions are automatically satisfied and the control law required to follow the given shaped trajectory is obtained from dynamic equations. Unlike traditional methods and similar to other shapebased approaches this method, does not require any numerical propagation and allows to work with a limited set of free parameters. Though this approach has already shown to be promising for the preliminary design of optimal interplanetary trajectories with multiple gravity assists manoeuvres, still an analysis of the optimality of the obtainable solutions is lacking. In particular its suitability to initialise indirect methods and direct collocation approaches. In the case of direct method the control provided by the pseudo-equinoctial element approach will be used to initialise the software code DITAN which makes use of a direct transcription by Finite Elements in Time. In the case of indirect methods controls and states computed with the shape-based approach will be used to compute the adjoint variables and the residuals on the optimality condition. Convergence properties will be shown for both methods and for two classes of optimization problems: minimum quadratic control problems and minimum final mass problems. Two test cases will be considered: a direct transfer from the Earth to an asteroid and a multiple revolution spiral.
\end{abstract}

